\title{
The thiol/disulfide balance in ketone positive and ketone negative pregnant women with nausea and vomiting - a prospective study in a tertiary center
}

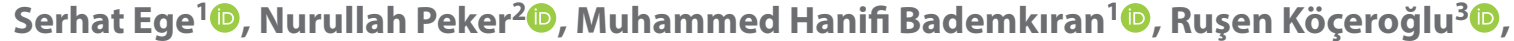

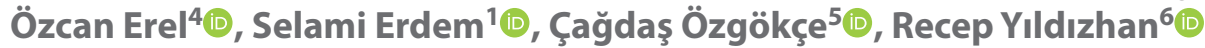 \\ ${ }^{1}$ Department of Gynecology and Obstetrics, Health Sciences University, Gazi Yasargil Training and Research Hospital, Diyarbakir, Turkey \\ ${ }^{2}$ Department of Gynecology and Obstetrics, Dicle University, Diyarbakır, Turkey \\ ${ }^{3}$ Department of Biochemistry, Health Sciences University, GaziYasargil Training and Research Hospital, Diyarbakir, Turkey \\ ${ }^{4}$ Department of Biochemistry, Yıldırım Beyazit University, Faculty of Medicine, Ankara, Turkey \\ ${ }^{5}$ Department of Gynecology and Obstetrics, Health Sciences University, Van Training and Research Hospital, Van, Turkey \\ ${ }^{6}$ Department of Gynecology and Obstetrics, Bilim University, Florance Nightingale Hospital, Istanbul, Turkey
}

\begin{abstract}
Objectives: We aimed to investigate the thiol/disulfide balance in ketone positive (hyperemesis gravidarum) and ketone negative pregnant women with nausea and vomiting.

Material and methods: A total of 60 patients under the $14^{\text {th }}$ week of pregnancy were included in this study, and they were divided into two groups. Group 1 included 30 pregnant women with ketone positive, group 2 included 30 ketone negative pregnant women with nausea, and vomiting.

Results: The native thiol, disulfide, and total thiol concentrations were measured using an automated method and compared among the two groups. There were also three indexes that are derived from disulfide, native and total thiol (Index $1=100 \times$ disulfide/native thiol); (Index $2=100 \times$ disulfide/total thiol); (Index $3=100 \times$ native thiol/total thiol). When compared with Group 1 and Group 2, total thiol was high, native thiol was low but not statistically significant. Disulphide $(p=0.046)$, index $1(p=0.036)$ and index $3(p=0.034)$ were statistically significant.

Conclusions: Patients with ketone positive are shifting to OS direction due to lack of nutrients and electrolytes. This study emphasizes the therapeutic potential of antioxidant supplementation, which is becoming an increasingly used approach in treating the symptoms of women with ketone positive.
\end{abstract}

Key words: hyperemesis gravidarum; nausea and vomiting; thiol/disulfide homeostasis; oxidative stress

Ginekologia Polska 2020; 91, 4: 207-209

\section{INTRODUCTION}

Nausea and/or vomiting is a common problem for pregnant women affecting approximately $80 \%$ of all pregnant women [1]. Hyperemesis gravidarum (HG), which is a severe form of nausea and/or vomiting, is diagnosed with ketonuria and more than $5 \%$ weight loss and occurs in $0.3-3 \%$ of all pregnant women $[2,3]$.

Thiols containing sulfhydryl groups [(R-SH)] are antioxidants that play an important role in the elimination of reactive oxygen species (ROS) that occur during oxidative stress (OS) [4]. With the separation of hydrogen from natural thiols, disulfide bonds [(R-S-S-R)] are formed, and with the release of this hydrogen, excess oxygen is bound in the environment and the cells are protected from oxidative damage. Conversely, disulfides can turn into native thiols, which is called dynamic thiol/disulfide homeostasis $[5,6]$.

In this study, we aimed to investigate the thiol/disulfide balance in ketone positive and ketone negative pregnant women with nausea and/or vomiting.

\section{MATERIAL AND METHODS}

A prospective study was performed with a total of 60 women with a history of nausea and/or vomiting, normal body mass index $\left(20-25 \mathrm{~kg} / \mathrm{m}^{2}\right)$ and aged between 
18-35 years at the department of obstetrics and gynaecology. Patients were evaluated in two groups: Group 1 consisted of 30 patients with ketone positive (HG). Group 2 consisted of 30 patients having nausea and/or vomiting more than three times per day during pregnancy and did not have ketonuria.

Patients with trophoblastic diseases, diabetes, preeclampsia, thyroid diseases, inflammatory diseases, kidney diseases, liver diseases, smoking or alcohol habits and pregnancies older than 14 weeks were excluded from the study. Gestational weeks were determined by the last menstrual period and ultrasonography.

Blood samples were taken and centrifuged for $10 \mathrm{~min}$ utes at 4,000 rpm. The serum portion was separated and stored at $-80^{\circ} \mathrm{C}$ until assessment of thiol/disulfide homeostasis.

Serum thiol/disulfide homeostasis was evaluated with a new and automated measurement technique by Erel [7]. Thiol/disulfide homeostasis results were calculated $\mu \mathrm{mol} / \mathrm{L}$.

The study protocol was approved by a regional committee (number 3 ).

\section{Statistical analysis}

All statistical analyses were done with SPSS 20.0 (IBM, New York, USA), MS Office Excel. The continuous variables were expressed as median (min-max); categorical variables were expressed as $\mathrm{n}(\%)$. The difference of continuous variables between groups was calculated by the Mann Whitney U-test. $p \leq 0.05$ was regarded as significant.

\section{RESULTS}

There were also three indexes that are derived from disulfide, native and total thiol (Index $1=100 \times$ disulfide/nativethiol); Index $2=100 \times$ disulfide/total thiol); Index $3=100 \times$ native thiol/total thiol). When compared with Group 1 and Group 2; total thiol was high, native thiol was low but not statistically significant. Disulphide $(p=0.046)$, index $1(p=0.036)$ and index $3(p=0.034)$ were statistically significant (Tab. 1).

\section{DISCUSSION}

Dynamic thiol/disulfide homeostasis is an essential predictor of OS in our body. Strengthening the enzyme mechanism and immune system depends on changes in thiol/disulfide balance $[8,9]$. It is thought that HG is caused by the imbalance between free ROS that are formed by OS and antioxidants, and it has been found that antioxidant balance was low andthe OS index was higher in the HG group [10]. Previously OS has been investigated in $\mathrm{HG}$ and healthy pregnant women. However, ketone positive and ketone negative pregnant women were compared for the first time. In this study, we investigated $O S$ related changes due to malnutrition by compar- ing thiol/disulfide balance between ketone positive and ketone negative pregnant women with nausea and/or vomiting. The findings were in favor of OS in ketone positive patients.

There are strong relationships between HG and OS [4]. First, a decrease in the antioxidant system is observed as a result of insufficient intake of vitamins and other energy sources taken as a result of reduced nutrition [11,12]. Many antioxidant enzymes have been shown to decrease due to HG. Another important mechanism is the increase in the synthesis of ROS in the presence of $\mathrm{H}$. pylori [13].

Onaran et al. [14] and Güney et al. [15] found a deficiency in the antioxidant system in HG. There is limited information about the importance of antioxidant effect in early fetus development. However, it increases the importance of antioxidants to free radical damage later in the fetus [16].

Ketonuria shows its carnage at the adipose site as a marker for HG, and it is widely used as HG-defined. Due to this carnage, there is correlation between the degree of ketonuria and HG [17]. While there is a negative correlation between disease severity and native thiol and total thiol, a positive correlation is detected between disulfide [18]. The American College of Obstetricians and Gynecologists (ACOG) currently recommends that a combination of oral pyridoxine hydrochloride (vitamin B6) and doxylamine succinate be used as first-line treatment for nausea and/or vomiting during pregnancy if pyridoxine monotherapy does not relieve symptoms [19]. Ege et al. [20] it has been shown that malnutrition in HG patients suppresses the antioxidant system and consequently the immune system is affected. Based on our results, in additional supplementa-

\begin{tabular}{|c|c|c|c|}
\hline & $\begin{array}{l}\text { Grup } 1 \\
(n=30)\end{array}$ & $\begin{array}{l}\text { Grup } 2 \\
(n=30)\end{array}$ & $\begin{array}{l}\text { p* } \\
\text { value }\end{array}$ \\
\hline & \multicolumn{3}{|c|}{ median (min-max) } \\
\hline Age [years] & $28(18-34)$ & $28(20-35)$ & 0.672 \\
\hline $\begin{array}{l}\text { Fasting Glucose } \\
{[\mathrm{mg} / \mathrm{dL}]}\end{array}$ & $94(73-123)$ & $85.5(66-122)$ & 0.139 \\
\hline Native Thiol $[\mu \mathrm{mol} / \mathrm{L}]$ & $\begin{array}{l}598.7 \\
511.3-695.6)\end{array}$ & $\begin{array}{l}627.7 \\
(510.9-772.1)\end{array}$ & 0.062 \\
\hline $\begin{array}{l}\text { Total Thiol } \\
\text { [Umol/L] }\end{array}$ & $\begin{array}{l}632.3 \\
(556.5-740.4)\end{array}$ & $\begin{array}{l}655.4 \\
(536.1-802.3)\end{array}$ & 0.089 \\
\hline Disulfide & $21(11.4-45.3)$ & $19.3(12.2-26.8)$ & 0.048 \\
\hline Index 1 & $3.51(1.89-8.45)$ & $2.87(1.76-4.61)$ & 0.036 \\
\hline Index 2 & $3.33(1.82-7.23)$ & $2.82(1.7-23)$ & 0.082 \\
\hline Index 3 & $\begin{array}{l}93.35 \\
85.54-96.36)\end{array}$ & $\begin{array}{l}94.58 \\
(91.56-96.61)\end{array}$ & 0.034 \\
\hline
\end{tabular}

Index $1-100 \times$ disulfide/native thiol; Index $2-100 \times$ disulfide/total thiol Index $3-100 \times$ native thiol/total thio

*Mann-Whitney-U test 
tion of - SH-containing compounds can be discussed in ketone positive group.

The limitation of this study was the small number of patients having particitated. Secondly, we were unable to measure the pre-pregnancy thiol/disulfide status of the forementioned pregnant women. Finally, we did not look at the level of H.pylori that causes of HG.

\section{CONCLUSIONS}

In conclusion, patients with HG are shifting to OS direction due to lack of nutrients and electrolytes. A significant difference between the ketone positive and ketone negative groups shows that all nausea and/or vomiting will not cause $O S$ and that the body can tolerate OS by considering a precise balance. This study emphasizes the importance of therapeutic support in the treatment of symptoms in ketone positive patients, where antioxidant supplements are gaining importance day by day.

\section{REFERENCES}

1. Wijayasinghe $S$, Bentvelzen $A$, Guenot $C$, et al. [Nausea and vomiting in pregnancy]. Rev Med Suisse. 2018; 14(614): 1397-1400, indexed in Pubmed: 30091330.

2. London V, Grube S, Sherer DM, et al. Hyperemesis Gravidarum: A Review of Recent Literature. Pharmacology. 2017; 100(3-4): 161-171, doi: 10.1159/000477853, indexed in Pubmed: 28641304.

3. Austin K, Wilson K, Saha S. Hyperemesis Gravidarum. Nutr Clin Pract. 2019; 34(2): 226-241, doi: 10.1002/ncp.10205, indexed in Pubmed: 30334272 .

4. Fait V, Sela S, Ophir E, et al. Hyperemesis gravidarum is associated with oxidative stress. Am J Perinatol. 2002; 19(2): 93-98, doi: 10.1055/s-200223554, indexed in Pubmed: 11938483.

5. Fait V, Sela S, Ophir E, et al. Peripheral polymorphonuclear leukocyte priming contributes to oxidative stress in early pregnancy. J Soc Gynecol Investig. 2005; 12(1): 46-49, doi: 10.1016/j.jsgi.2004.08.005, indexed in Pubmed: 15629671.

6. Prakash M, Shetty MS, Tilak P, et al. Total Thiols: Biomedical importance and their alteration in various disorders. Online Journal of Health and Allied Sciences. ; 2009.
7. Erel O, Neselioglu S. A novel and automated assay for thiol/disulphide homeostasis. Clin Biochem. 2014; 47(18): 326-332, doi: 10.1016/j.clinbiochem.2014.09.026, indexed in Pubmed: 25304913.

8. Biswas S, Chida AS, Rahman I. Redox modifications of protein-thiols: emerging roles in cell signaling. Biochem Pharmacol. 2006; 71(5): 551-564, doi: 10.1016/j.bcp.2005.10.044, indexed in Pubmed: 16337153.

9. Circu ML, Aw TY. Reactive oxygen species, cellular redox systems, and apoptosis. Free Radic Biol Med. 2010; 48(6): 749-762, doi: 10.1016/j. freeradbiomed.2009.12.022, indexed in Pubmed: 20045723.

10. Aksoy H, Aksoy AN, Ozkan A, et al. Serum lipid profile, oxidative status, and paraoxonase 1 activity in hyperemesis gravidarum. J Clin Lab Anal. 2009; 23(2): 105-109, doi: 10.1002/jcla.20298, indexed in Pubmed: 1928845.

11. Celik F, Guzel Al, Kuyumcuoglu U, et al. Dietary antioxidant levels in hyperemesis gravidarum: a case control study. Ginekol Pol. 2011; 82(11): 840-844, indexed in Pubmed: 22384617.

12. van Stuijvenberg ME, Schabort I, Labadarios $D$, et al. The nutritional status and treatment of patients with hyperemesis gravidarum. Am J Obstet Gynecol. 1995; 172(5): 1585-1591, doi: 10.1016/0002-9378(95)90501-4, indexed in Pubmed: 7755076.

13. Cevrioglu AS, Altindis M, Yilmazer $M$, et al. Efficient and non-invasive method for investigating Helicobacter pylori in gravida with hyperemesis gravidarum: Helicobacter pylori stool antigen test. J Obstet Gynaecol Res. 2004; 30(2): 136-141, doi: 10.1111/j.1447-0756.2003.00173.x, indexed in Pubmed: 15009618.

14. Onaran $\mathrm{Y}$, Kafali $\mathrm{H}$, Duvan $\mathrm{Cl}$, et al. Relationship between oxidant and antioxidant activity in hyperemesis gravidarum. J Matern Fetal Neonatal Med. 2014;27(8):825-828, doi: 10.3109/14767058.2013.842549, indexed in Pubmed: 24041031.

15. Güney M, Oral B, Mungan T. Serum lipid peroxidation and antioxidant potential levels in hyperemesis gravidarum. Am J Perinatol. 2007; 24(5): 283-289, doi: 10.1055/s-2007-981429, indexed in Pubmed: 17514602.

16. Perkins AV. Endogenous anti-oxidants in pregnancy and preeclampsia. Aust N Z J Obstet Gynaecol. 2006; 46(2): 77-83, doi: 10.1111/j.1479-82 8X.2006.00532.x, indexed in Pubmed: 16638026.

17. Bashiri A, Neumann L, Maymon E, et al. Hyperemesis gravidarum: epidemiologic features, complications and outcome. Eur J Obstet Gynecol Reprod Biol. 1995; 63(2): 135-138, doi: 10.1016/0301-2115(95)02238-4, indexed in Pubmed: 8903768.

18. Ergin M, Cendek BD, Neselioglu S, et al. Dynamic thiol-disulfide homeostasis in hyperemesis gravidarum. J Perinatol. 2015; 35(10): 788-792, doi: 10.1038/jp.2015.81, indexed in Pubmed: 26156064.

19. ACOG. Practice Bulletin: nausea and vomiting in pregnancy. Obstetrics and Gynecology. ; 2004.

20. Ege $\mathrm{S}$, Bademkiran $\mathrm{MH}$, Peker $\mathrm{N}$, et al. Evaluation of catalase, myeloperoxidase and ferroxidase values in pregnant women with hyperemesis gravidarum. Ginekol Pol. 2019; 90(11): 651-655, doi: 10.5603/GP.2019.0110, indexed in Pubmed: 31802466 